\title{
Pertussis: increasing disease as a consequence of reducing transmission
}

Ricardo Águas, Guilherme Gonçalves, M Gabriela M Gomes

Lancet Infect Dis 2006; 6: $\mathrm{xx}-\mathrm{xx}$ RÁ, GG, and MGMG are at the Instituto Gulbenkian de Ciência, Oeiras, Portugal. Correspondence to: Dr M Gabriela M Gomes, Instituto Gulbenkian de Ciência, Apartado 14, 2781-901 Oeiras, Portugal. Tel +351 214 464626; fax +351214407970 ggomes@igc.gulbenkian.pt

Since the 1980s, the occurrence of pertussis cases in developed countries has increased and shifted towards older age groups. This resurgence follows 30 years of intense mass vaccination, and has been attributed primarily to three factors: (1) more effective diagnosis of the disease, (2) waning of vaccine-induced immunity, and (3) loss of vaccine efficacy due to the emergence of new Bordetella pertussis strains. Here we develop and analyse a mathematical model to assess the plausibility of these hypotheses. We consider that exposure to $B$ pertussis through natural infection or vaccination induces an immune response that prevents severe disease but does not fully prevent mild infections. We also assume that these protective effects are temporary due to waning of immunity. These assumptions, describing the mode of action of adaptive immunity, are combined with a standard transmission model. Two distinct epidemiological scenarios are detected: under low transmission, most infections lead to severe disease; under high transmission, mild infections are frequent, boosting clinical immunity and maintaining low levels of severe disease. The two behaviours are separated by a reinfection threshold in transmission. As a result, the highest incidence of severe disease is expected to occur at intermediate transmission intensities-near the reinfection threshold—suggesting that pertussis resurgence may be induced by a reduction in transmission, independently of vaccination. The model is extended to interpret the outcomes of current control measures and explore scenarios for future interventions.

\section{Introduction}

Pertussis is a highly infectious bacterial disease caused by Bordetella pertussis. Pertussis is one of the ten most common causes of death from infectious disease worldwide, accounting for 300 000-400 000 deaths each year. ${ }^{1}$ The manifestations of $B$ pertussis vary with the host, symptoms ranging from the most typical cough outbursts with inspiratory whoop, periods of apnoea, and post-tussive vomiting, to symptoms so mild as to be confounded with those of a common cold. B pertussis is increasingly recognised as a principle cause of persistent cough in adults. ${ }^{2}$

The introduction of mass vaccination of children in the mid-20th century decreased the incidence of pertussis so enormously as to make it an uncommon disease. Estimates for individual efficacy of pertussis vaccines are around $70-80 \%$ and induced protection is of limited duration. ${ }^{3-5}$ Despite high vaccination coverage, $B$ pertussis remains endemic and reports of increasing incidence in the USA, ${ }^{6}$ Canada, ${ }^{7}$ the Netherlands, Australia, ${ }^{9}$ France, ${ }^{10}$ the UK, ${ }^{11}$ and Poland have been accumulating since the $1980 \mathrm{~s} .{ }^{12}$ This increase has been accompanied by a shift to older age groups ${ }^{13-15}$ raising concerns about household transmission to vulnerable infants $^{16,17}$ and calling for a reappraisal of clinical management in adolescents and adults ${ }^{18,19}$ and a reinforcement of vaccination strategies. ${ }^{20}$ The problem stimulates further epidemiological research ${ }^{21-23}$ and mathematical modelling. ${ }^{24-26}$

The resurgence of pertussis has been the subject of considerable debate. ${ }^{27,28}$ Hypotheses to explain increased reporting in developed countries have focused mainly on three aspects: (1) increased recognition of the disease in adolescent and adults, ${ }^{22,29}$ (2) waning of vaccine-induced immunity, ${ }^{2,21,30}$ and (3) loss of vaccine efficacy due to an antigenic shift of $B$ pertussis. ${ }^{31}$
The first hypothesis postulates that increasing notifications reflect an intensification of the reporting system. Attempts to demonstrate that the altered patterns are due to notification trends alone, however, have so far been unsuccessful. 15,18,21,32 The other two views argue for changes induced by widespread vaccine use. On the one hand, the waning of vaccine-induced immunity is likely to shift the incidence to older age groups, but this does not explain the increase in total incidence. Furthermore, since estimates for the duration of vaccine-induced immunity are in the range of 5-10 years, it remains unclear how its introduction could be responsible for an epidemiological change 30 years later. Antigenic shift and adaptation of $B$ pertussis to the vaccine with its consequent loss of efficacy, on the other hand, is not consistent with the data across countries, being evident in the Netherlands ${ }^{31}$ but not in France, ${ }^{33}$ for example.

Here we propose an alternate explanation based on two main observations: first, immunity has a limited effect on transmission of infection and a greater effect on severity of disease; ${ }^{1,22,34,35}$ second, immunity wanes over time..$^{5,36,37}$ We encapsulate these assumptions into a mathematical model and conclude that a peak in the incidence of severe disease is attained at intermediate transmission. As a result, increased disease and increased age at infection may be caused simultaneously by reducing $B$ pertussis transmission. As illustrated in figure 1 , this happens when transmission is above the reinfection threshold. ${ }^{38,39}$

The reinfection threshold has a dramatic influence on overall epidemiology, creating a clear distinction between two epidemiological scenarios. Whereas below the threshold the levels of overall infection are low, with most cases being primary infections likely to lead to severe disease, above the threshold transmission is 
strong enough to overcome immunity and, as such, mild infections are frequent, boosting clinical immunity and maintaining low levels of severe disease. These effects are independent of vaccination. On this basis, we propose that the reported increases in severe pertussis incidence patterns may be due to a reduction in transmission occurring independently of vaccine action. Many factors might account for a reduction in transmission in developed countries over the past 25 years. These factors range from improvements in general health-care provision and socioeconomic conditions to demographic processes such as the general decreasing trends in birth rates observed consistently in developed countries in the second half of the 20th century.

\section{Minimal model}

The model structure is defined by classifying the population according to $B$ pertussis infection and immunity, and representing the rates of change as transmission progresses (figure 2). In a broad sense, the model distinguishes between infections in an immunologically naive individual, whose level of protection is minimal, and infections in an individual with a previously primed response either by infection or vaccination. In the first instance, and in the interest of clarity, it is assumed that natural infection and vaccination induce similar immune protection that is lost at similar rates. ${ }^{36}$ The results extend, however, to the more realistic scenario of vaccine-induced immunity waning faster ${ }^{5}$ (see web appendix). The model is designed to explore the implications of reinfection on pertussis epidemiology. Reinfection can potentially occur in individuals with maximum levels of protection, resulting in mild or atypical symptoms,,$^{13,16,37}$ and these events change from rare to very common as transmission increases across the reinfection threshold. ${ }^{38,39}$

Individuals are born at a rate $\mu$ into compartment $B$, where they are protected from infection. Birth and death rates are assigned as to provide an average lifespan of 70 years. Protection of individuals in $B$ can be due to reduced exposure and this effect is lost at a rate $\gamma$. A proportion, $v$, is vaccinated and transferred to $R$ (partly immune), while the remaining, 1-v, become susceptible, $S$. The latter are subject to a force of infection, $\lambda$, and develop severe pertussis with typical symptoms when infected, $I_{1}$. Individuals mount an immune response and recover at a rate $\tau$ into compartment $R$. The recovery rate is set to provide an average duration of infection of approximately 2 weeks. Although the symptoms of pertussis last about 2 months overall, individuals are mostly infectious during that catarrhal stage that typically takes place during the first 2 weeks of infection. Individuals in $R$ have clinical immunity but are, nevertheless, still subject to reinfection, moving to compartment $I_{2}$ if transmission is

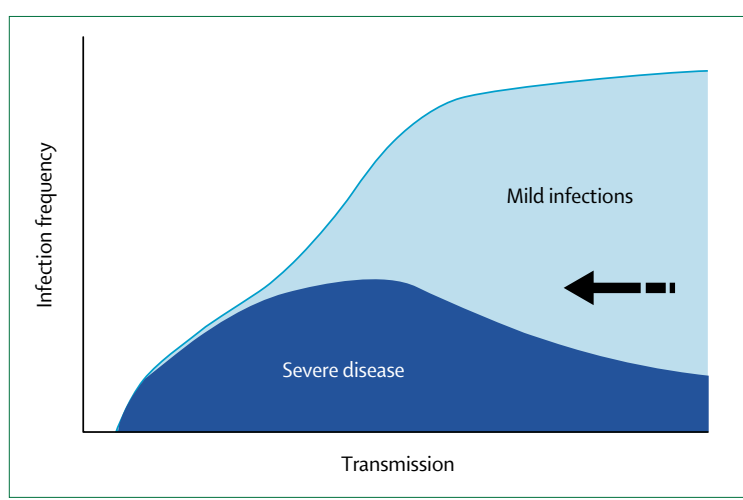

Figure 1: Scheme representing increased disease incidence as a consequence of reducing transmission

The dark blue area refers to the proportion of infections that result in severe disease and the light blue area represents the contribution of mild infections to the total number of infections. Reducing transmission is illustrated by the arrow.

intense enough. Reinfections from $R$ occur at a reduced rate, $\sigma \lambda$, and we use $\sigma=0 \cdot 25$, which is consistent with estimates of vaccine efficacy. These infections are mild or atypical, but likely to have a crucial role in maintaining a level of partial immunity that prevents the occurrence of severe disease.

Severe $\left(I_{1}\right)$ and mild $\left(I_{2}\right)$ infections are distinguished by the severity of symptoms and infectiousness-we consider that individuals in $I_{1}$ are twice as infectious as those in $I_{2}$. During a mild infection, although individuals have an atypical form of disease that can easily be

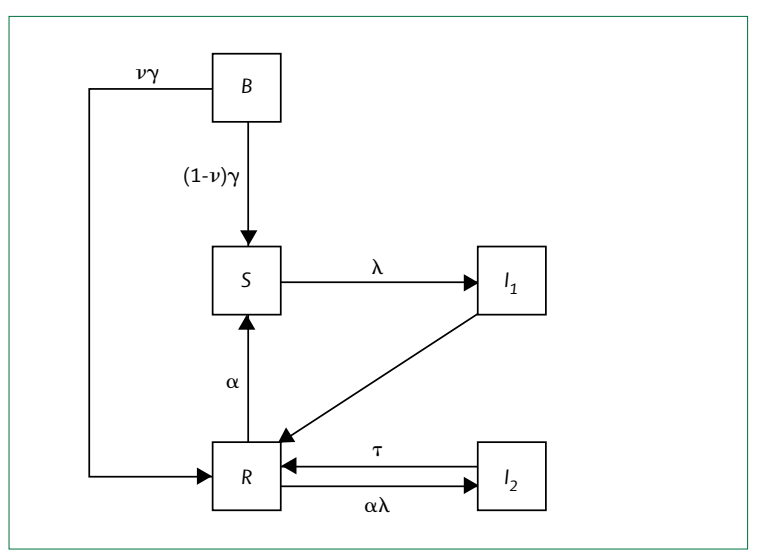

Figure 2: Model for the $B$ pertussis transmission dynamics

Five epidemiological classes are organised into two major groups. The uninfected (three compartments on the left) are classified as follows. $B=$ protected for a short period after birth; $R=$ recovered from infection or vaccinated, with partial immunity; $S=$ susceptible after losing protection conferred in $B$ or $R$. The infected (two compartments on the right) are classified as: $I_{1}=$ severe disease resulting from exposure of susceptible individuals; and $\mathrm{I}_{2}=$ mild infection resulting from exposure of recovered individuals. The parameters are described in the table, and the model equations are: $\delta B / \delta t+\delta B / \delta a=-(\gamma+\mu) B ;$

$\delta S / \delta t+\delta S / \delta a=(1-v) \gamma B+\alpha R-(\gamma+\mu) S$

$\delta R / \delta t+\delta R / \delta a=v \gamma \gamma+\tau\left(l_{1}+I_{2}\right)-(\sigma \lambda+\alpha+\mu) R_{i}$

$\delta I_{1} / \delta t+\delta I_{1} / \delta a=\lambda S-(\tau+\mu) I_{1}$

$\delta I_{2} / \delta t+\delta I_{2} / \delta a=\sigma \lambda S-(\tau+\mu) I_{2}$

The boundary conditions at age $a=0$ are $B(t, 0)=\mu$ and $S(t, 0)=R(t, 0)=I_{1}(t, 0)=$ $I_{2}(t, 0)=0$. 


\begin{tabular}{|lll|}
\hline Parameter & Definition & Value \\
\hline$\mu$ & Birth and death rate & $1 / 70$ years $^{-1}$ \\
$\gamma$ & Rate of loss of protection after birth & 2 years $^{-1}$ \\
$\tau$ & Rate of recovery from infection & 24 years $^{-1}$ \\
$\alpha$ & Rate of loss of acquired immunity & $1 / 20$ years $^{-1}$ \\
$\sigma \mid$ & Relative rate of mild/severe infections & $0 \cdot 25$ \\
$\beta$ & Transmission coefficient & Variable \\
$\eta$ & Relative infectiousness of mild/severe infections & $0 \cdot 5$ \\
$\omega$ & Vaccination coverage & $95 \%$ \\
\hline Table: Model parameters & \\
\hline
\end{tabular}

confounded with other upper respiratory infections, they have the capability of transmitting $B$ pertussis. The broad spectrum of clinical presentations of pertussis poses serious difficulties to the precise determination of the parameters that characterise the infectious states, but the accuracy of these estimates is not critical given the generic tendencies of our results. Although it is possible to estimate a period of about 2 months for the resolution of pertussis symptoms, it is nonetheless important to consider that individuals are especially infectious during the catarrhal stage that takes place in the first 2 weeks of infection. Therefore, we use a recovery rate of 2 weeks. Individuals in $R$ lose their immunity at a rate $\alpha$, unless it is boosted by reinfection. This rate is assumed so that clinical immunity lasts on average 20 years, which is consistent with empirically determined values of waning immunity. ${ }^{6-15}$

The rate of infection, $\lambda$, is considered proportional to the frequency of infectious individuals, $\lambda=\beta\left(I_{1}+\eta I_{2}\right)$, where $\eta$ represents the infectiousness of mild infections relative to severe infections. For the purpose of illustration we set this parameter to $\eta=0 \cdot 5$, but this value is not critical to the results. The constant of proportionality, $\beta$, is designated the "transmission coefficient" and varies between populations. The basic reproduction number, $R_{0}$, is proportional to $\beta$, and their relation is given by $R_{0}=\beta /(\tau+\mu)$. This parameter represents the number of secondary infections that an index case is expected to generate in a totally susceptible population, and is a crucial measure in setting targets for disease elimination. All parameters are summarised in the table.

\section{Results}

Changes in infectious disease epidemiology are often attributed to thresholds in transmission, and these [Au: these what?] are detected through observed trends and comparative studies. We consider the transmission coefficient (or $R_{0}$ ) as an independent parameter and describe the importance of this measure on the epidemiology.

Analysing the proportion of infectious individuals at equilibrium as a function of the basic reproduction number, we can identify two evident transmission thresholds (figure 3A). The epidemic threshold indicates the transmission coefficient required for infection to invade a susceptible population and is realised when $R_{0}=1$. The reinfection threshold, at $R_{0}=1 / \sigma \eta$, represents the transmission coefficient above which reinfection dominates the dynamics. ${ }^{38,39}$ This [Au: crossing the reinfection threshold?] is associated with a major increase in mild infections $\left(I_{2}\right)$ and consequent

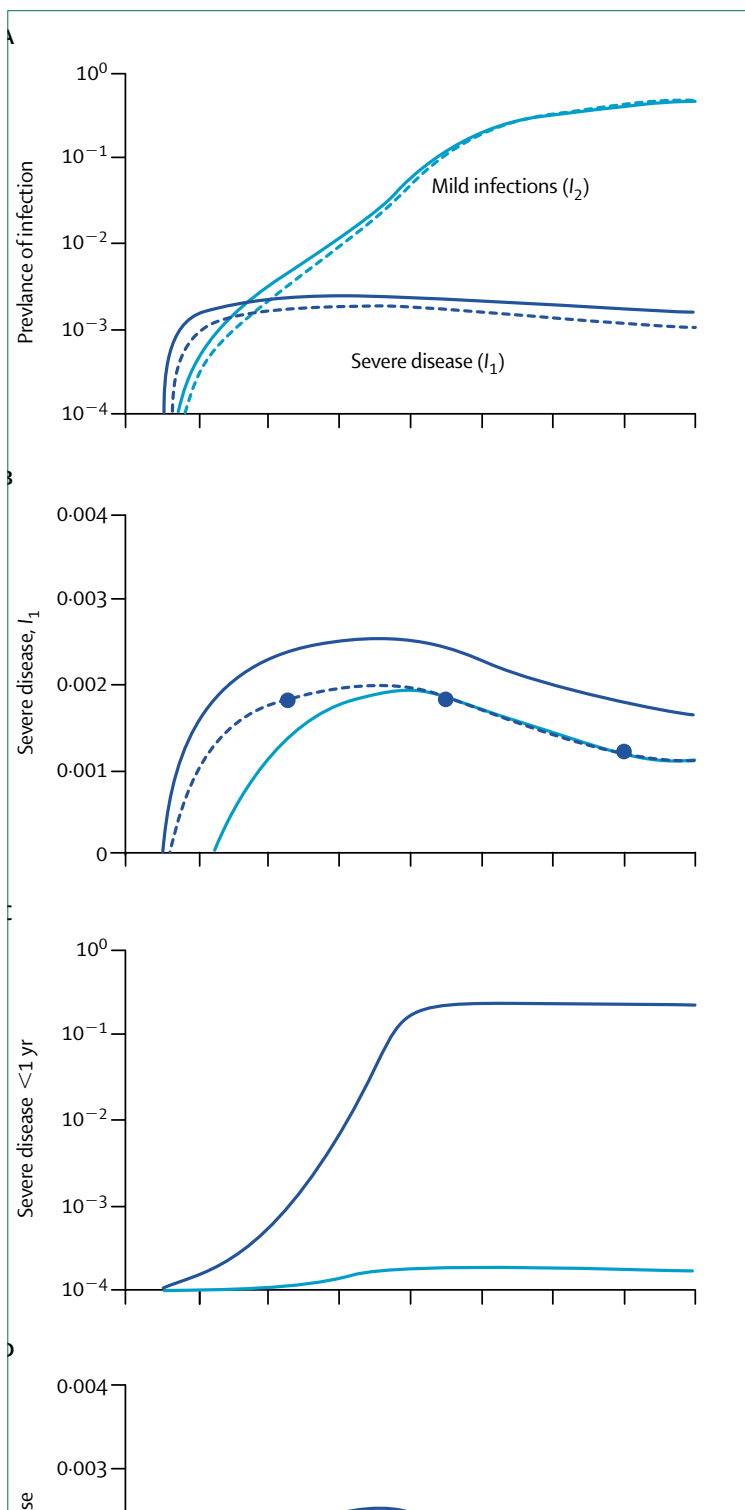

Figure 3: Peak in severe disease at intermediate transmission and the impact of vaccination [Au: units for $y$-axes?]

(A) Proportion infected at equilibrium (in logarithmic scale) without vaccination (full lines) and with 95\% childhood vaccination (broken lines). The thin lines are mild infections $\left(I_{2}\right)$, and heavy lines are severe disease $\left(I_{1}\right)$. (B) Severe disease burden (in linear scale) without vaccination (full line), with $95 \%$ childhood vaccination (broken line), and an extension to boosting vaccination of adolescents and adults at 10-year intervals on average (broken line). [Au: what do circles indicate?] (C) Proportion of severe disease in children under 1 year without vaccination (full line) and with $95 \%$ childhood vaccination (broken line). (D) Proportion of severe disease without vaccination (full line) and with $95 \%$ childhood vaccination with a vaccine that confers long lived protection against disease. 
boosting of immunity. As a result, there is a noticeable decrease in severe disease $\left(I_{1}\right)$ as transmission increases above the reinfection threshold. Altogether, these epidemiological processes induce a peak in the incidence of severe disease at intermediate transmission-ie, near the reinfection threshold (more evident in figure $3 \mathrm{~B}$ ).

The key underlying processes are sketched by focusing on the possible fates of individuals who have been previously infected or vaccinated, and retain a level of partial immunity. These individuals are subject to two competing rates: they either loose their immunity, or they are subject to a mild infection that boosts their immunity. The epidemiological outcome is very sensitive to which of the two processes dominates the dynamics. Waning of immunity is dominant below the reinfection threshold and underlies the trend of increasing disease with transmission. Boosting of immunity becomes dominant above the reinfection threshold, and underlies the less intuitive trend of decreasing disease with increasing transmission. In the limiting situations of very high transmission settings, only susceptible newborns have an episode of severe disease and the adult population acts as a reservoir.

The impact of childhood vaccination on the incidence of severe disease is substantial throughout all values of $R_{0}$, especially in the younger age groups (figure 3C). Mild infections, by contrast, are practically insensitive to vaccination when transmission is above the reinfection threshold, maintaining the partly immune status of the population, but simultaneously posing major obstacles to further control (dotted lines in figure 3B). This observation has important implications for the debated issue of introducing boosting vaccination of adolescents and adults in 10-year intervals as a strategy to maintain lifelong immunity. ${ }^{20,28}$ The model analysed here suggests that such strategy has serious limitations when transmission is above peak incidence, but this first insight should be followed by more detailed models based on more detailed data. As such, boosting vaccination should not be discouraged by these results, but rather taken as a unique opportunity to do cohort studies and learn more about the dynamics of immunity to pertussis.

We extended the model to test the potential of improving vaccines as to induce longer protection (the model extension described in the web appendix). Above the reinfection threshold, where control is a greater challenge, increasing the duration of protective immunity can lead to major reductions in severe disease, despite not having a substantial impact on infection overall (figure 3D). Comparison with figure 3B suggests that the effectiveness of long lasting immunity is not easily reproduced by repeated boosting, because above the reinfection threshold the levels of transmission are constantly high, effectively hitting individuals whose immunity dropped below protective levels before the next booster.

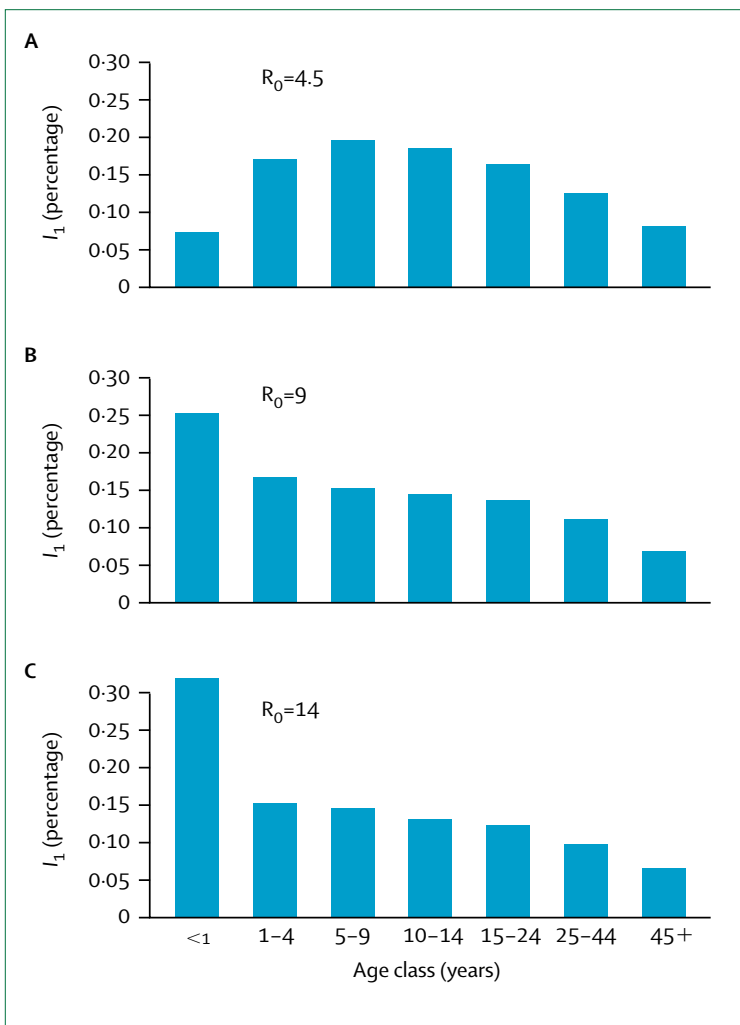

Figure 4: Age profiles of the annual incidence of severe disease obtained for the model populations marked by dots in figure $3 \mathrm{~B}$

Age profiles of reported cases are important indicators of infectious disease transmission. We simulated a cohort using the force of infection at each of the three equilibria marked as dots in figure 3B, under a childhood vaccination programme with $95 \%$ coverage. The resulting age profiles are represented in figure 4. For $R_{0}=4 \cdot 5$, which is well below previous estimates for pertussis $^{40}$ and below the peak incidence for our model, the profile shows an increase in incidence from infants to older children. At $R_{0}=9$, which is above the peak incidence, the burden of severe disease is approximately the same as for $R_{0}=4 \cdot 5$, but the age profile gives higher incidence in infants, thus showing a reversed trend. When transmission is at $R_{0}=14$, the overall incidence of disease is lower and the average age at infection is also lower. The trends in pertussis epidemiology that motivated this work are therefore simulated [Au: ok?] by reducing transmission from $R_{0}=14$ to $R_{0}=9$, for example. The simultaneous occurrence of increasing disease incidence and a shift to older age groups, which conflicts with traditional epidemiological models, is here linked to the role of waning and partial immunity in regulating reinfection.

\section{Discussion}

Epidemiological changes provide unique opportunities to investigate fundamental aspects of infection and 
disease. The accumulating reports of increased pertussis incidence in developed countries and the associated shift to older age groups have been calling for an explanation. Pertussis is elusive. Behind a small number of reported cases of severe typical pertussis, there is a vast infectious process where most cases are mild and atypical reinfections of partly immune individuals remain unreported. The epidemiology of clinical pertussis cannot be detached from this wider context. Because $B$ pertussis infection is mostly unreported, its epidemiology is a subject of animated debate and controversy.

Given the level of uncertainty, we constructed a mathematical model based on two assumptions regarding immunity and transmission that are mostly consensual: immunity has a limited effect on transmission but a substantial effect on the severity of disease, and immunity wanes over time. Model analysis identifies a peak in severe disease at intermediate levels of transmission. As the potential for transmission increases, the incidence of severe disease initially increases. However, this trend is reversed when the socalled reinfection threshold is crossed and, from then on, severe disease decreases as transmission is further increased. On this basis, we propose that pertussis transmission occurs above the reinfection threshold and that increases in incidence reported in developed countries result directly from reducing transmission. A similar idea has been proposed for malaria ${ }^{41}$ and may be more generally applicable to diseases where immunity has a limited effect on transmission of infection and a greater effect on disease severity. The mechanism also requires that these effects wane over time.

The waning of immunity continually replenishes the susceptible pool, thus becoming a serious obstacle to control even with regular boosting of the adult population through vaccination. Regular boosting will raise individual immunity, but this protective effect will gradually decrease until the next boosting. This vaccination strategy is unlikely to eliminate the wide variation in immunity and thus in severity of symptoms unless vaccination effectively interrupts transmission or provides uninterrupted protection against disease. Interruption of transmission can be achieved below the reinfection threshold but not above. That would require a vaccine that confers more protection than that conferred by infection. As for uninterrupted protection, the ideal would be a vaccine that could provide life-long protection. The closest option is regular boosting with vaccines that provide shorter-lived protection. The model described here suggests that the ups and downs in immunity inherent to such strategy, together with its inability to interrupt transmission above the reinfection threshold, make effectiveness very uncertain. Given that high levels of transmission will be maintained, disease will strike at any opportunity, and this is supported by more elaborate models ${ }^{24}$ and clinical trials. ${ }^{30}$ However, if transmission is somehow reduced to levels below the reinfection threshold, there will be a substantial decrease in disease incidence as a result of boosting vaccination of adolescents and adults. Overall, our results do not indicate that boosters given as a part of the recommended vaccination schedule would be useless or in any case harmful. On the contrary, boosters will provide unique opportunities to monitor the dynamics of immunity to pertussis through specially designed cohort studies. The resulting knowledge would be extremely valuable to intervention design and could provide important insights into vaccine research.

The most optimistic scenarios were explored by analysing the potential impact of vaccines that could be more protective than natural immunity. Two improvements were implemented: one induced stronger protection against reinfection and the other increased the duration of protection. Improving vaccines to induce longer protection is the best option. Even under high transmission, increasing the duration of protective immunity can lead to major reductions in disease incidence, despite not having a substantial impact on infection overall.

Mathematical models of infectious diseases are standard tools in public health. They can sharpen our understanding of the specific aspects of infectious disease dynamics, and can be extended to assess the possible impact of alternative control strategies. Moreover, models can be used to compare different hypotheses and generate new ones. The minimal model presented here integrates $B$ pertussis transmission with disease incidence. The mode of action of immunitypartly protective and waning-was identified as the basis for all the results. The simplicity of this model facilitates the clarity of interpretation, but makes extreme conclusions unavoidable. Therefore, this initial insight should be taken as a hypothesis to be followed by more detailed models and observational studies.

Conflicts of interest

[Au: please declare any conflicts of interest]

\section{Acknowledgments}

We thank António Coutinho and Natalia Mantilla-Berniers for valuable comments. This research was funded by the Calouste Gulbenkian Foundation (FCG), the Portuguese Research Council (FCT), and the European Commission, grant MEXT-CT-2004-14338.

References

1 Crowcroft NS, Stein C, Duclos P, Birmingham M. How best to estimate the global burden of pertussis? Lancet Infect Dis 2003; 3: 413-18.

2 Gilberg S, Njamkepo E, Parent du Châtelet I, et al. Evidence of Bordetella pertussis infection in adults presenting with persistent cough in a French area with very high whole-cell vaccine coverage. J Infect Dis 2002; 186: 415-18.

3 Taranger J, Trollfors B, Bergfors E, et al. Immunologic and epidemiologic experience of vaccination with a monocomponent pertussis toxoid vaccine. Pediatrics 2001; 108: 115-20.

4 Jefferson T, Rudin M, DiPietrantonj C. Systematic review of the effects of pertussis vaccines in children. Vaccine 2003; 21: 2003-14.

5 Wirsing von König CH, Halperin S, Riffelmann M, Guiso N Pertussis of adults and infants. Lancet Infect Dis 2002; 2: 744-50. 
6 Güris D, Strebel PM, Bardenheier, et al. Changing epidemiology of pertussis in the United States: increasing reported incidence among adolescents and adults, 1990-1996. Clin Infect Dis 1999; 28 1230-37.

7 Ntezayabo B, De Serres G, Duval B. Pertussis resurgence in Canada largely caused by a cohort effect. Pediatr Infect Dis $J$ 2003; 22: 22-27.

8 de Melker HE, Coyn-van Spaendonck MAE, Rümke HC, et al. Pertussis in the Netherlands: an outbreak despite high levels of immunization with whole cell vaccine. Emerg Infect Dis 1997; 3: 175-78.

9 Andrews R, Herceg A, Roberts C. Pertussis notifications in Australia, 1991 to 1997. Commun Dis Intell 1997; 21: 145-48.

10 Baron S, Njamkepo E, Grimprel E, et al. Epidemiology of pertussis in French hospitals in 1993 and 1994: thirty years after a routine use of vaccination. Pediatr Infect Dis J 1998; 17: 412-18.

11 van Buynder PG, Owen D, Vurdien JE, Andrews NJ, Matthews RC, Miller E. Bordetella pertussis surveillance in England and Wales: 1995-7. Epidemiol Infect 1999; 123: 403-11.

12 Gzyl A, Augustynowicz E, Rabczenko D. Pertussis in Poland [Au: journal?] 2004; 33: 358-65.

13 Christie C, Marx ML, Marchant CD, Reising SF. The 1993 epidemic of pertussis in Cincinnati - resurgence of disease in a highly immunized population of children. N Engl J Med 1994; 331: $16-21$.

14 Skowronski DM, De Serres G, MacDonald D, et al. The changing age and seasonal profile of pertussis in Canada. J Infect Dis 2002; 185: 1448-53.

15 de Melker HE, Schellekens JFP, Neppelenbroek SE. Reemergence of pertussis in the highly vaccinated population of the Netherlands: observations on surveillance data. Emerg Infect Dis 2000; 6: 348-57.

16 Wirsing von König CH, Postels-Multani S, Bock HL, Schmitt HJ. Pertussis in adults: frequency of transmission after household exposure. Lancet 1995; 346: 1326-29.

17 Crowcroft NS, Britto J. Whooping cough-a continuing problem. BMJ 2002; 324: 1537-38.

18 De Serres G, Shadmani R, Duval B, et al. Morbidity of pertussis in adolescents and adults. J Infect Dis 2000; 182: 174-79.

19 Hewlett EL, Edwards KM. Pertussis—not just for kids. N Engl J Med 2005; 352: 1215-22.

20 Forsyth KD, Campins-Marti M, Caro J, et al. New pertussis strategies beyond infancy: recommendations by the Global Pertussis Initiative. Clin Infect Dis 2004; 39: 1802-09.

21 Black S. Epidemiology of pertussis. Pediatr Infect Dis J 1997; 16: S85-89.

22 Pebody RG, Gay NJ, Giammanco A, et al. The seroepidemiology of Bordetella pertussis infection in western Europe. Epidemiol Infect 2005; 133: 159-71.

23 Préziosi MP, Yam A, Wassilak SG, et al. Epidemiology of pertussis in a west African community before and after introduction of a widespread vaccination program. Am J Epidemiol 2002; 155: 891-96.
24 Hethcote H. Simulations of pertussis epidemiology in the United States: effects of adult booster vaccinations. Math Biosci 1999; 158: 47-73.

25 van Boven M, de Melker H, Schellekens J, Kretzschmar M. A model based evaluation of the 1996-7 pertussis epidemic in the Netherlands. Epidemiol Infect 2001; 127: 73-85.

26 van Boven M, Ferguson N, van Rie A. Unveiling the burden of pertussis. Trends Microbiol 2004; 12: 116-19.

27 Cherry JD. The science and fiction of the "resurgence" of pertussis. Pediatrics 2003; 112: 405-06.

28 De Serres G, Duval B. Pertussis vaccination beyond childhood. Lancet 2005; 365: 1015-16.

29 Yih W, Lett S, des Vignes F, Garrison K, Sipe P, Marchant C. The increasing incidence of pertussis in Massachusetts adolescents and adults, 1989-1998. J Infect Dis 2000; 182: 1409-16.

30 Grimprel E, Baron S, Lévy-Bruhl D, et al. Influence of vaccination coverage on pertussis transmission in France. Lancet 1999; 354: 1699-700.

31 Mooi F, van Loo I, King A. Adaptation of Bordetella pertussis to vaccination: a cause for its reemergence? Emerg Infect Dis 2001; 7: 526-28.

32 Torvaldsen S, McIntyre P. Do variations in pertussis notifications reflect incidence or surveillance practices? A comparison of infant notification rates and hospitalization data in NSW. N S W Public Health Bull 2003; 14: 81-84.

33 Weber C, Boursaux-Eude C, Coralie G, Caro V, Guiso N. Polymorphism of Bordetella pertussis isolates circulating for the last 10 years in France, where a single effective whole-cell vaccine has been used for more than 30 years. J Clin Microbiol 2001; 39: 4396-403.

34 Fine PE, Clarkson JA. The recurrence of whooping cough: Possible implications for assessment of vaccine efficacy. Lancet 1982; 1: 666-69.

35 Broutin H, Guégan J-F, Elguero E, Simondon, Cazelles B. Largescale comparative analysis of pertussis population dynamics: periodicity, synchrony, and impact of vaccination. Am J Epidemiol 2005; 161: 1159-67.

36 Cherry JD. Epidemiological, clinical, and laboratory aspects of pertussis in adults. Clin Infect Dis 1999; 28: S112-17.

37 Versteegh FGA, Schellekens JFP, Nagelkerke AF, Roord JJ. Laboratory-confirmed reinfections with Bordetella pertussis. Acta Paediatr 2002; 91: 95-99.

38 Gomes MGM, White LJ, Medley GF. Infection, reinfection, and vaccination under suboptimal immune protection: epidemiological perspectives. J Theor Biol 2004; 228: 539-49.

39 Gomes MGM, White LJ, Medley GF. The reinfection threshold. $J$ Theor Biol [Au: published yet?] 2005 (in press).

40 Anderson RM, May RM. Infectious diseases of humans: dynamics and control. New York: Oxford University Press; 1991.

41 Snow RW, Marsh K. The consequences of reducing Plasmodium falciparum transmission in Africa. Adv Parasitol 2002; 52: 235-64. 